Corresponding authors: moelling@molgen.mpg.de, felixbroecker@gmx.net

(C) 2015 Broecker et al. This article is distributed under the terms of the Creative Commons

Attribution License, which permits unrestricted reuse and redistribution provided that the original author and source are credited.

Ontology terms: recurrent infection of the gastrointestinal tract

Published by Cold Spring Harbor Laboratory Press

doi: $10.1101 /$ mcs.a000448

\section{Long-term changes of bacterial and viral compositions in the intestine of a recovered Clostridium difficile patient after fecal microbiota transplantation}

\author{
Felix Broecker, ${ }^{1,2,6,7}$ Jochen Klumpp, ${ }^{3,7}$ Markus Schuppler, ${ }^{3}$ Giancarlo Russo, ${ }^{4}$ \\ Luc Biedermann, ${ }^{5}$ Michael Hombach, ${ }^{1}$ Gerhard Rogler, ${ }^{5}$ and Karin Moelling ${ }^{1,2}$ \\ ${ }^{1}$ Institute of Medical Microbiology, University of Zurich, 8006 Zurich, Switzerland; ${ }^{2}$ Max Planck Institute for \\ Molecular Genetics, 14195 Berlin, Germany; ${ }^{3}$ Institute of Food, Nutrition and Health, ETH Zurich, 8096 Zurich, \\ Switzerland; ${ }^{4}$ Functional Genomics Center Zurich, University of Zurich and ETH Zurich, 8057 Zurich, Switzerland; \\ ${ }^{5}$ Division of Gastroenterology and Hepatology, University Hospital Zurich, 8006 Zurich, Switzerland
}

Abstract Fecal microbiota transplantation (FMT) is an effective treatment for recurrent Clostridium difficile infections (RCDIs). However, long-term effects on the patients' gut microbiota and the role of viruses remain to be elucidated. Here, we characterized bacterial and viral microbiota in the feces of a cured RCDI patient at various time points until $4.5 \mathrm{yr}$ post-FMT compared with the stool donor. Feces were subjected to DNA sequencing to characterize bacteria and double-stranded DNA (dsDNA) viruses including phages. The patient's microbial communities varied over time and showed little overall similarity to the donor until 7 mo post-FMT, indicating ongoing gut microbiota adaption in this time period. After $4.5 \mathrm{yr}$, the patient's bacteria attained donor-like compositions at phylum, class, and order levels with similar bacterial diversity. Differences in the bacterial communities between donor and patient after $4.5 \mathrm{yr}$ were seen at lower taxonomic levels. C. difficile remained undetectable throughout the entire timespan. This demonstrated sustainable donor feces engraftment and verified long-term therapeutic success of FMT on the molecular level. Full engraftment apparently required longer than previously acknowledged, suggesting the implementation of year-long patient follow-up periods into clinical practice. The identified dsDNA viruses were mainly Caudovirales phages. Unexpectedly, sequences related to giant algae-infecting Chlorella viruses were also detected. Our findings indicate that intestinal viruses may be implicated in the establishment of gut microbiota. Therefore, virome analyses should be included in gut microbiota studies to determine the roles of phages and other viruses-such as Chlorella viruses -in human health and disease, particularly during RCDI.

\section{INTRODUCTION}

Clostridium difficile is the leading cause of hospital-acquired infectious diarrhea (Lo Vecchio and Zacur 2012). This bacterium capitalizes on antibiotic disruption of the normal microbiota to colonize the intestine, causing disease mainly by secreted toxins (Lo Vecchio and Zacur 2012). Recent years have seen an increase in lethal $C$. difficile infections and the emergence

${ }^{6}$ Present address: Max Planck Institute of Colloids and Interfaces, 14424 Potsdam, Germany.

${ }^{7}$ These authors contributed equally to this work. 
COLD SPRING HARBOR Molecular Case Studies
Long-term human microbiota after fecal transplant

of strains with increased toxin production and antibiotic resistance (McDonald et al. 2005; Warny et al. 2005; Razavi et al. 2007; Lessa et al. 2012).

The failure of antibiotics to fully eliminate $C$. difficile leads to recurrent disease episodes in $\sim 30 \%$ of patients (Johnson 2009). This fueled the investigation of fecal microbiota transplantation (FMT) as an alternative treatment option, whereby patients are instilled with healthy donor feces to replenish intestinal microbiota that prevent the growth of $C$. difficile. FMT has shown impressive success rates of $~ 90 \%$ against RCDIs and no severe adverse effects (Gough et al. 2011; Cammarota et al. 2014; O'Horo et al. 2014). A recent controlled clinical trial demonstrated the superiority of FMT to antibiotics for RCDI treatment (van Nood et al. 2013). FMT led to increased donor-like intestinal bacterial diversities within 2 wk (van Nood et al. 2013). Knowledge about the long-term effects of FMT, however, is presently not available. In addition, previous studies mainly focused on bacteria. Because viruses, especially phages, are the most abundant intestinal entities with the ability to influence microbial communities (Barr et al. 2013; Virgin 2014), they may well be relevant to C. difficile infection and the microbial changes following FMT. This is also suggested by recent findings that phages play a causative role in inflammatory bowel disease (IBD), which, similar to RCDI, is characterized by pathologically altered gut microbiota (Norman et al. 2015).

We recently reported on a recovered RCDI patient whose fecal bacteria were of chimeric composition of the patient and the healthy sister donor up to 7 mo post-FMT (Broecker et al. 2013), suggesting that stable attainment of intestinal microbial communities may take longer time periods. Here, we followed up this patient until $4.5 \mathrm{yr}$ post-FMT, characterized fecal bacterial communities by $16 \mathrm{~S}$ rRNA gene sequencing at various time points, and further analyzed previously reported viromes (Broecker et al. 2013) in comparison to the donor.

\section{RESULTS}

\section{Patient History}

Details on the patient history have been published (Broecker et al. 2013). Briefly, the female patient was 51 years old when admitted to the University Hospital of Zurich with her sixth episode of RCDI, suffering from severe diarrhea and weight loss. Stool samples tested positive for $C$. difficile by standard toxin A and B immunoassays, selective agar cultures, and agglutination assays. The first episode of $C$. difficile infection occurred $2 \mathrm{yr}$ before hospital admission after 7 mo of multiple antibiotic treatment against a complicated jawbone infection.

Multiple rounds of treatments against RCDI with the recommended antibiotics metronidazole and vancomycin, partly in conjunction with the probiotic Saccharomyces cerevisiae, were only temporally successful but lead to recurrence after cessation. A therapeutic trial with intravenous immunoglobulins did not induce significant clinical responses. Finally, FMT was performed with donor feces from the patient's sister that tested negative for a variety of bacterial and viral pathogens. A suspension of donor feces in sterile $0.9 \%$ sodium chloride solution was applied intra-anally to the patient. Before the treatment, the patient was given vancomycin to suppress growth of pathogenic $C$. difficile as well as loperamide to prevent diarrhea. Following FMT, the patient reported changes in bowel movements and intermittent obstipation, both of which ceased within 10 wk. Ever since, the patient has remained free of symptoms for almost $5 \mathrm{yr}$ now.

\section{Bacterial Communities of the Patient Were Highly Variable up to 7 mo Post-FMT and Similar to the Donor after $4.5 \mathrm{yr}$ at the Phylum Level}

A total of six fecal samples of the patient after FMT and the stool donor were collected at various time points and subjected to $16 \mathrm{~S}$ rRNA gene sequencing (Fig. 1A). The specimens 
A
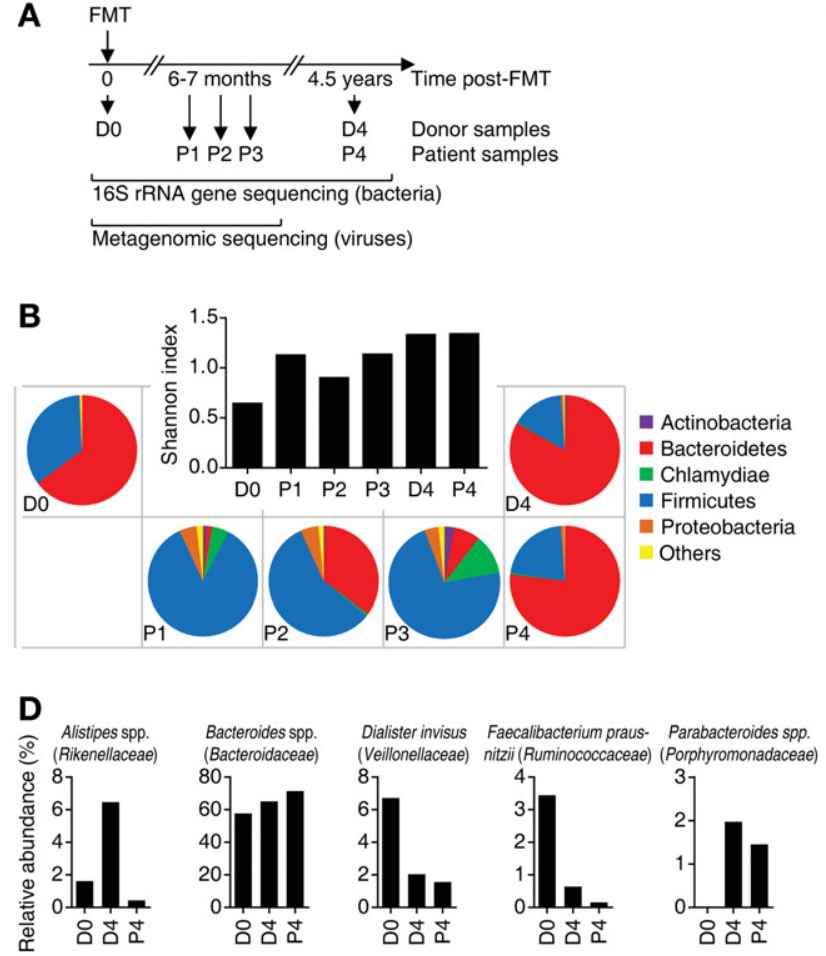

E

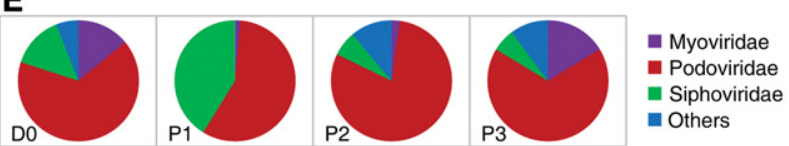

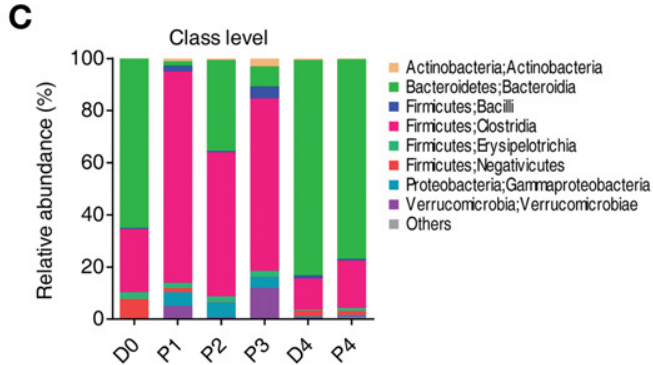
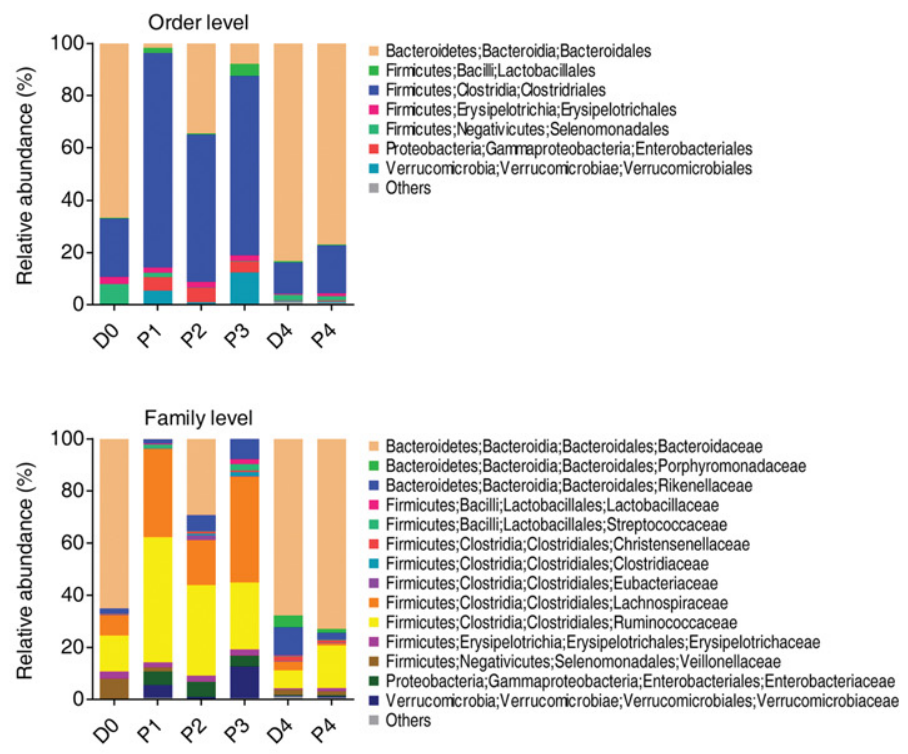

Figure 1. Analysis of fecal microbiota. (A) Sample description. Fecal samples of the donor and the patient were collected at the indicated time points and subjected to metagenomic and/or 16S rRNA gene sequencing. FMT, fecal microbiota therapy. (B) Bacterial compositions at the phylum level are shown as pie charts. The inlay graph shows bacterial diversities inferred by Shannon indices as bars. (C) Bacterial compositions at the class, order, and family levels (from top to bottom) are shown as stacked bar graphs. Only taxa supported by at least $1 \%$ of total reads at each level are shown. (D) Relative abundances of the five most dominant bacterial genera in samples D0, D4, and P4 are shown as bar graphs. Dialister and Faecalibacterium genera were solely represented by the indicated species in each sample. The respective family names are given in parentheses. (E) Viral compositions of Caudovirales families are shown as pie charts.

included two long-term samples of the donor and the patient (D4 and P4, respectively), both collected $4.5 \mathrm{yr}$ post-FMT. Four additional samples, one donor sample at the time of FMT (D0) and three patient samples 6-7 mo after the treatment (P1-P3), have been previously analyzed by metagenomic sequencing (Broecker et al. 2013). In the present study, targeted 16S rRNA gene sequencing was also applied to the latter samples to make them comparable to samples D4 and P4. A summary of the sequencing yields and accuracies is provided in Table 1.

Taxonomic analysis of the donor samples at the time of FMT (D0) and $4.5 \mathrm{yr}$ later (D4) revealed bacterial communities dominated by the Bacteroidetes phylum (D0: 65\% and D4: 83\%), followed by Firmicutes (34\% and 15\%) (Fig. 1B). Remaining bacteria accounted for $<2 \%$ in these two samples. In contrast, the patient samples P1-P3 collected 6-7 mo post-FMT were mainly composed of up to $85 \%$ (P1) of phylum Firmicutes. In this timespan, bacterial communities underwent extensive fluctuations. For instance, 


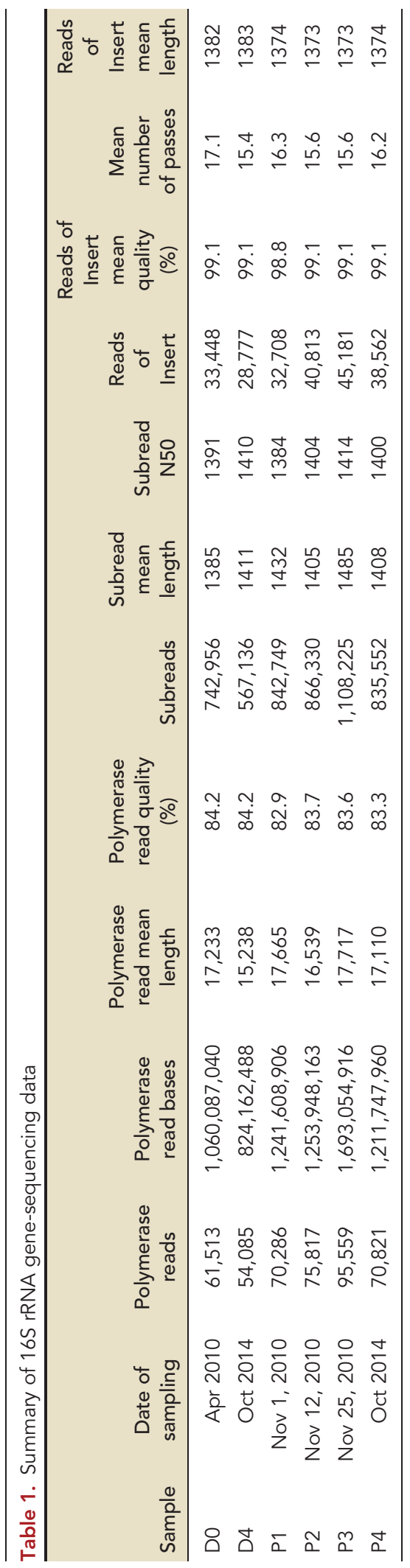


Bacteroidetes comprised 2\% (P1), then 35\% (P2), and finally 8\% (P3) of all bacteria. The phylum Chlamydiae, barely detectable in the donor, constituted up to $12 \%$ (P3) of the patient's bacteria. In contrast to P1-P3, the patient sample $4.5 \mathrm{yr}$ post-FMT (P4) mainly contained Bacteroidetes (77\%) followed by Firmicutes $(21 \%)$ and $<2 \%$ remaining bacteria.

Bacterial diversities were estimated by calculating the Shannon diversity indices for all samples at the species level. The Shannon indices showed a high degree of variability even in the healthy donor, where an about twofold increase from the time point of FMT (D0) to $4.5 \mathrm{yr}$ afterward (D4) was observed (Fig. 1B). In the patient samples, a trend toward increasing diversity from the time period covered by samples P1-P3 to $4.5 \mathrm{yr}$ post-FMT (P4) was observed that may, however, have been due to the fact that samples P1-P3 could not be fully resolved down to the species level. At this time point, the bacterial diversities in the samples from patient (P4) and donor (D4) were highly similar.

\section{Bacterial Communities of the Patient Were Similar to Those of the Donor after $4.5 \mathrm{yr}$ up to the Order Level but Showed Differences at Lower Taxonomic Levels}

More detailed insights into the bacterial communities were gained at lower taxonomic levels. In all six samples, bacteria of the Bacteroidetes phylum were exclusively assigned to the order Bacteroidales (Bacteroidia class) (Fig. 1C). The Firmicutes phylum stratified into the four orders Lactobacillales (Bacilli class), Clostridiales (Clostridia class), Erysipelotrichales (Erysipelotrichia class), and Selenomonadales (Negativicutes class). At the order level, bacterial communities of D4 and P4 remained highly similar.

At the family level, some differences between these two samples became apparent. For instance, the families Porphyromonadaceae and Rikenellaceae, both of the Bacteroidales order as well as the Lachnospiraceae family of the Clostridiales order, were more abundant in D4 than in P4.

Only samples D0, D4, and P4 were compared in more detail at the genus level, because samples P1-P3 provided only full taxonomic information up to the family level. The relative abundances of the five most dominant genera in samples D0, D4, and P4, including two that were only represented by a single species, are shown in Figure 1D. Fractions of Bacteroides spp., Dialister invisus, and Parabacteroides spp. were roughly similar between samples D4 and P4, whereas Alistipes spp. and Faecalibacterium prausnitzii were more abundant in D4. Most interestingly, we did not identify any sequences assigned to $C$. difficile species in any of the analyzed samples (data not shown).

\section{Communities of dsDNA Viruses Were Variable and Consisted Mainly of Caudovirales Phages}

The analysis of viral dsDNA sequences reported earlier (Broecker et al. 2013) revealed the presence of 22 viruses throughout samples D0, P1, P2, and P3 (Table 2). In each sample, eight to 11 different viruses were identified, mainly belonging to the Caudovirales order (tailed dsDNA phages) that contains the viral families Myo-, Podo-, and Siphoviridae. Most viruses, 14 of 22, were identified uniquely in either sample. Three phages, the Erwinia phage vB_EamP-L1 (Podoviridae) and the two Bacteroides phages B124-14 and B40-8 (Siphoviridae), were consistently detected in all four samples and each contained phages of all three Caudovirales groups. Of these, Podoviridae were consistently most abundant. Myo- and Siphoviridae showed highly variable abundances (Fig. 1E). The patient sample P3 contained sequences related to the Paramecium bursaria Chlorella virus 1 (PBCV-1) that infects eukaryotic algae (Table 2; Van Etten 2003). 
Table 2. List of identified intestinal viruses

\begin{tabular}{|c|c|c|c|c|c|c|c|c|}
\hline & Virus & $\begin{array}{l}\text { NCBI nucleotide } \\
\text { accession number }\end{array}$ & $\begin{array}{l}\text { Genome } \\
\text { size (bp) }\end{array}$ & $\begin{array}{c}\text { Number of } \\
\text { annotated } \\
\text { ORFs }\end{array}$ & $\begin{array}{c}\text { Present } \\
\text { in D0? }\end{array}$ & $\begin{array}{l}\text { Present } \\
\text { in } \mathrm{P} 1 ?\end{array}$ & $\begin{array}{l}\text { Present } \\
\text { in } \mathrm{P} 2 ?\end{array}$ & $\begin{array}{l}\text { Present } \\
\text { in P3? }\end{array}$ \\
\hline \multirow[t]{6}{*}{ Myoviridae } & Enterobacteria phage RB16 & NC_014467.1 & 176,788 & 271 & Yes & & & Yes \\
\hline & Enterobacteria phage RB43 & NC_007023.1 & 180,500 & 292 & Yes & & & Yes \\
\hline & Klebsiella phage KP15 & NC_014036.1 & 174,436 & 258 & & & & Yes \\
\hline & Bacillus phage BCР78 & NC_018860.1 & 156,176 & 227 & Yes & & & \\
\hline & Bacillus phage SP10 & NC_019487.1 & 143,986 & 236 & & & Yes & \\
\hline & Streptococcus phage EJ-1 & NC_005294.1 & 42,935 & 73 & & Yes & & \\
\hline \multirow[t]{3}{*}{ Podoviridae } & Erwinia phage vB_EamP-L1 & NC_019510.1 & 39,282 & 51 & Yes & Yes & Yes & Yes \\
\hline & Escherichia phage TL-2011b & NC_019445 & 44,784 & 57 & & Yes & Yes & Yes \\
\hline & Bacillus phage $\phi 29$ & NC_011048 & 19,282 & 27 & & & Yes & \\
\hline \multirow[t]{8}{*}{ Siphoviridae } & Bacteroides phage B124-14 & NC_016770 & 47,159 & 68 & Yes & Yes & Yes & Yes \\
\hline & Bacteroides phage B40-8 & NC_011222 & 44,929 & 46 & Yes & Yes & Yes & Yes \\
\hline & Clostridium phage $\phi \mathrm{CP} 34 \mathrm{O}$ & NC_019508 & 38,309 & 52 & & yes & & \\
\hline & $\begin{array}{l}\text { Lactococcus phage } 936 \\
\text { sensu lato }\end{array}$ & KC182544 & 27,302 & 49 & & Yes & & \\
\hline & Lactococcus phage $\phi 41$ & n.a. & n.a. & n.a. & & Yes & & \\
\hline & Listeria phage 2389 & n.a. & n.a. & n.a. & Yes & & & \\
\hline & Listeria phage B025 & NC_009812.1 & 42,653 & 65 & Yes & & & \\
\hline & $\begin{array}{l}\text { Rhodococcus phage } \\
\text { ReqiPepy6 }\end{array}$ & NC_023735 & 76,797 & 107 & Yes & & & \\
\hline $\begin{array}{l}\text { Unclassified } \\
\text { Caudovirales }\end{array}$ & Sinorhizobium phage PBC5 & NC_003324 & 57,416 & 83 & Yes & & Yes & Yes \\
\hline Unclassified & Clostridium phage D-1873 & n.a. & n.a. & n.a. & & & Yes & Yes \\
\hline phages & Tetrasphaera phage TJE1 & NC_019930 & 49,219 & 66 & & & & Yes \\
\hline $\begin{array}{l}\text { Unclassified } \\
\text { dsDNA }\end{array}$ & $\begin{array}{l}\text { Paramecium bursaria } \\
\text { Chlorella virus } 1\end{array}$ & NC_000852.5 & 330,611 & 802 & & & & Yes \\
\hline viruses & Clostridium phage $\phi \mathrm{SM} 101$ & NC_008265.1 & 38,092 & 53 & Yes & & & \\
\hline
\end{tabular}

NCBI, National Center for Biotechnology Information; ORFs, open reading frames; n.a., not available.

\section{DISCUSSION}

FMT has been shown to be a promising treatment option for RCDI patients that leads to replenishment of the patients' gut microbiota through application of donor feces (Gough et al. 2011; van Nood et al. 2013; Cammarota et al. 2014; O'Horo et al. 2014). Here, we investigated the long-term effects of FMT by analyzing fecal microbiota of a cured RCDI patient in comparison to the donor until $4.5 \mathrm{yr}$ after the procedure.

To analyze the bacterial compositions, we chose 16S rRNA gene sequencing using SMRT (single-molecule real-time) sequencing on the Pacific Biosciences (PacBio) platform. Despite its known limitations regarding raw read quality, this sequencing method has been successfully used previously to resolve compositions of microbial communities (Marshall et al. 2012; Fichot and Norman 2013). In the present study, we used the latest chemistry (C6) that reinforces the main characteristics that make the PacBio platform attractive for microbial sequencing: the absence of sequence context-dependent error and GC-coverage biases (Carneiro et al. 2012; Quail et al. 2012) and, most importantly, the read length. As shown in Table 1, the generated reads were long enough to accurately cover full-length $16 \mathrm{~S}$ rDNA fragments through the application of the Reads of Insert protocol, an update of the old circular consensus sequence (CCS). The resulting reads were first used as a query against the Silva ribosomal RNA gene database (Quast et al. 2013) using the BLAST algorithm (Altschul et al. 1990). Then, the MEGAN program (Huson et al. 2007) was used to build 
taxonomic landscapes of the bacterial communities. This workflow has been successfully used before to study microbial communities (Thomas et al. 2012; Li et al. 2013; Sharpton 2014) even of high diversity (Valverde and Mellado 2013). In the present study, both the high-quality scores of the Reads of Insert of $>99 \%$ and the mean number of passes of at least 15.4 indicated reliable sequencing results (Table 1).

The bacterial composition of the donor was relatively stable and comparable at the time of FMT and $4.5 \mathrm{yr}$ later (Fig. 1B), which is in accordance with the known temporal stability of adult intestinal microbiota (Zoetendal et al. 1998). At the phylum level, Bacteroidetes were most prominent, followed by Firmicutes, typical of healthy gut microbiota (Eckburg et al. 2005). The patient's fecal microbiota underwent extensive compositional fluctuations and were dominated by Firmicutes up to 7 mo post-FMT, suggesting ongoing adaptation processes of donor microbiota in the patient's intestine that may also reflect changes in nutrition over the observation period. This is in accordance with our and other groups' recent findings that showed high degrees of bacterial variation in RCDI patients up to 7 mo post-FMT (Broecker et al. 2013; Weingarden et al. 2015). However, 4.5 yr post-FMT, the patient's bacteria have attained a donor-like composition at the phylum level, indicating full and stable engraftment of the donor's microbiota. The similarities between the donor's and patient's bacterial compositions remained up to the order level (Fig. 1C). Differences observed at lower taxonomic levels might reflect host-dependent adaptation processes or temporal fluctuations. Of note, four of the five most prominent genera identified in both donor samples as well as the patient sample after $4.5 \mathrm{yr}$, Alistipes, Bacteroides, Dialister, and Faecalibacterium (Fig. 1D), are known constituents of healthy fecal microbiota (Claesson et al. 2011; Joossens et al. 2011). This further indicated that FMT led to healthy and sustainable microbiota in the patient. Parabacteroides, another genus typical of healthy fecal microbiota (Claesson et al. 2011) was identified in both long-term samples but not in the donor at the time of FMT, perhaps reflecting temporal fluctuations in the healthy donor. One notable species detected in these three samples is Faecalibacterium prausnitzii (Fig. 1D). This species was also detected in the patient samples 6-7 mo post-FMT with abundances of $<0.1 \%$ (data not shown). Faecalibacterium prausnitzii is recognized as one of the most important species of healthy individuals and normally constitutes $>5 \%$ of the gut microbiota (Miquel et al. 2013). Lower than usual levels of F. prausnitzii have been associated with Crohn's disease (Sokol et al. 2008; Joossens et al. 2011). The low abundance of F. prausnitzii in the samples of the present study, especially of the patient, is intriguing. However, this may still reflect normal fluctuations, as there have not been any symptoms of Crohn's disease or other apparent complications in the patient.

The fact that the patient's clinical symptoms, which included severe diarrhea in the absence of antibiotic treatment against C. difficile (Broecker et al. 2013), resolved promptly after FMT suggests that gut microbiota were able to exert normal metabolic functions even before full engraftment. This may be explained by the fact that the patient's bacterial diversity even during the highly variable time period up to 7 mo post-FMT was already in the range of the healthy donor. In agreement with the absence of symptoms until today, C. difficile bacteria were undetectable in the samples of the patient, similar to the donor who tested negative for $C$. difficile before FMT (Broecker et al. 2013). This showed sustainable elimination of $C$. difficile bacteria from the patient's intestine and successful therapy on the molecular level. It is worth mentioning that the patient had to undergo two short-term antibiotic treatments for other indications with apparently no further consequences on the gut microbiota. The finding that the patient's fecal microbiota attained a highly donor-like composition after $4.5 \mathrm{yr}$ suggests that long-term follow-up should be implemented into clinical practice. Moreover, this finding highlights the importance of selecting donor feces with a healthy microbiota composition. An even better source material for FMT could be prospectively freeze-stored own feces. The suitability of frozen feces for FMT has been demonstrated 
COLD SPRING HARBOR Molecular Case Studies
Long-term human microbiota after fecal transplant

by a recent phase 1 clinical study, in which orally administered frozen capsules containing healthy fecal matter cured $90 \%$ of RCDI patients (Youngster et al. 2014).

Viral sequences were identified by metagenomic sequencing from the same DNA preparations that were used for $16 \mathrm{~S}$ sequencing. The analysis of viral dsDNA sequences from a previous study (Broecker et al. 2013) revealed the presence of Caudovirales phages in all investigated samples of the donor and the patient (Table 2). Caudovirales have been shown before to be the dominant viruses in the human intestine, followed by ssDNA phages of the Microviridae family that we were unable to detect with the metagenomic sequencing approach (Lepage et al. 2008; Norman et al. 2015). Three phages were identified in all of the analyzed samples of the donor and the patient. One is the Podovirus Erwinia phage VB_EamP-L1 that infects Erwinia amylovora bacteria, the causal agent of fire blight in Rosaceae species including apple and pear trees (Born et al. 2014). As its host, E. amylovora, is not a normal constituent of intestinal microbiota, this phage was likely a food contaminant that survived the stomach passage. The other two universally detected phages were the Siphoviruses Bacteroides phages B124-14 and B40-8 that infect bacteria of the Bacteroides genus abundantly found in the gut of healthy humans (Ogilvie et al. 2012). We detected low and variable quantities of the Bacteroides genus in the patient 6-7 mo post-FMT. In percent of total reads, $0.3 \%$ (P1), 20.7\% (P2), and 0.3\% (P3) were assigned to Bacteroides (data not shown). Even though $16 \mathrm{~S}$ rRNA gene sequencing did not fully resolve the genus level in these samples, they matched those of the Bacteroidaceae family that contained only Bacteroides spp. in samples D0, D4, and P4 (Fig. 1C). In contrast to P1-P3, both donor samples showed high abundances (57.7\% and $61.2 \%$, D0 and D4, respectively) of Bacteroides spp. similar to the patient sample $4.5 \mathrm{yr}$ post-FMT (71.4\%, P4) (Fig. 1D). The Bacteroides phages may have been transferred from the donor to the patient where they could have played a role in controlling Bacteroides populations during early stages through bacterial lysis, as suggested before (Lepage et al. 2008; Ogilvie et al. 2012; Norman et al. 2015).

The replication of phages is dependent on the presence of their bacterial host. Therefore, it is not surprising that we found extensive fluctuations in the virome when bacteria were also highly variable in the patient's samples up to 7 mo post-FMT. It has to be noted that the viral abundances presented in Figure 1E were produced by the metagenomic sequencing approach in which viruses with higher gene numbers could be overrepresented (Broecker et al. 2013). The Myoviridae may appear more abundant because of their larger average genomes and gene numbers (Table 2). The relatively small Podoviridae were therefore the most dominant Caudovirales phages among all samples. Phages are known to be able to regulate gut microbiota by bacterial lysis, horizontal gene transfer, and modulation of the intestinal immune system (Barr et al. 2013; Virgin 2014). It is thus tempting to speculate that they may have contributed to the bacterial population dynamics in the patient when the gut microbiota have not yet been fully established.

The identification of PBCV-1-related sequences in one of the patient's samples is intriguing because this virus has not yet been identified in the human intestine. PBCV-1 is a giant virus that contains about 800 open reading frames (ORFs), 400 protein-coding genes, and up to 16 tRNA genes. The related Acanthocystis turfacea Chlorella virus 1 (ATCV-1) has been identified recently in human nasopharyngeal samples where its presence correlated with reduced cognitive function (Yolken et al. 2014), showing that giant viruses may well be relevant for human health and disease. Sequences of Phycodnaviridae, the viral family harboring PBCV-1 and ATCV-1, have previously been reported to be present in the intestine of rodents (Phan et al. 2011). A possible role of PBCV-1 in the human intestine remains to be elucidated, but its presence may simply have resulted from the intake of Chlorella algaecontaminated freshwater (Van Etten 2003). The first identification of PBCV-1-related sequences in a human intestine, however, suggests that other unexpected viruses may be detected in future studies. 
COLD SPRING HARBOR Molecular Case Studies
Long-term human microbiota after fecal transplant
Phages are 10 -fold more abundant than their prokaryotic hosts in the human gut where they may influence bacterial diversity and population structure (Lepage et al. 2008; Minot et al. 2013). The low number of eight to 11 phages identified per sample here may be an underrepresentation, perhaps attributable to the DNA isolation procedure (Broecker et al. 2013). Also, the sequencing approach did not allow for distinguishing integrated prophages from genomes of free virus particles (Broecker et al. 2013). The isolation of viral genetic material from viral particles in stool supernatants may be more suitable to characterize fecal virus communities (Lepage et al. 2008; Phan et al. 2011; Norman et al. 2015). It has been reported that phages in the gut may be liberated under pathologic conditions, like inflammation (Lepage et al. 2008), suggesting that low numbers may correlate with healthy gut microbiota.

Overall, our findings demonstrate the long-term efficacy of FMT for the treatment of $\mathrm{RCDI}$ on the molecular level. Highly diverse phage communities suggest a possible role of phages during engraftment of donor microbiota. In light of a recent study that showed a causative role of phages in the etiology of IBD (Norman et al. 2015), gut viruses may be relevant during $C$. difficile disease and response to FMT as well. This is the subject of ongoing comprehensive investigations of fecal viromes of RCDI and IBD patients treated with FMT at the University Hospital and the ETH Zurich.

\section{METHODS}

\section{Metagenomic Sequencing}

The metagenomic-sequencing data used to characterize viruses in samples D0, P1, P2, and P3 (Table 1) is from a previous publication (Broecker et al. 2013). Briefly, DNA was isolated from $0.2 \mathrm{~g}$ of frozen-stool samples with the QIAamp DNA Stool Mini Kit (QIAGEN), then treated with DNase-free RNase (Fermentas), and further purified by phenol/chloroform extraction. Barcoded libraries were generated with the NEBNext DNA sample prep kit (New England Biolabs) and sequenced on an Illumina Genome Analyzer Ilx instrument in a 120base paired-end multiplex run. Read sets were assembled to contiguous sequences with the CLC Genomic Workbench V5. ORFs of these contigs were predicted with GLIMMER3 (Salzberg et al. 1998). The ORFs were queried in the NR-PROT protein database (Benson et al. 2009) using BLASTP (Altschul et al. 1990). The first listed protein hit of each ORF was taxonomically assigned using MEGAN. For more details, refer to Broecker et al. (2013).

\section{S rRNA Gene Sequencing}

Fecal DNA was isolated as described above in "Metagenomic Sequencing." For samples D0, P1, P2, and P3, the same DNA preparations used previously for metagenomic sequencing (Broecker et al. 2013) and stored at $-80^{\circ} \mathrm{C}$ were subjected to $16 \mathrm{~S}$ rRNA gene sequencing. A broad-range $16 \mathrm{~S}$ rDNA PCR was performed with the Phusion High-Fidelity PCR Master Mix (Finnzymes/NEB), using universal primers TPU-1 (AGAGTTTGATCMTGGCTCAG), 1387r (GGGCGGWGTGTACAAGGC), and one-tenth of primer Bif-8F (AGGGTTCGATTCTGGCT CAG) in order to amplify $\sim 1300 \mathrm{bp}$ of the bacterial $16 \mathrm{~S}$ rRNA genes. PCR cycling conditions were $3 \mathrm{~min}$ at $98^{\circ} \mathrm{C}\left(10 \mathrm{sec}\right.$ at $98^{\circ} \mathrm{C} 30 \mathrm{sec}$ at $55^{\circ} \mathrm{C}, 45 \mathrm{sec}$ at $\left.72^{\circ} \mathrm{C}\right) \times 24 ; 10 \mathrm{~min}$ at $\left.72^{\circ} \mathrm{C}\right)$. Sequencing on the PacBio RS II was performed with SMRT cell libraries prepared with the DNA Template Prep Kit 2.0 (250 bp to <3 kb) (Pacific Biosciences p/n 001-540-726) and 1400-bp selected target size. Consensus sequences were generated with the Reads of Insert protocol by retaining only sequences with $\geq 90 \%$ accuracy and $1400 \pm 100$ bp length. The minimum number of passes was set to 3 . A summary of the sequencing yields and accuracies is provided in Table 1. 
$\operatorname{CSH}^{\infty}$ C O L D S P R IN G H A R B OR Molecular Case Studies
Long-term human microbiota after fecal transplant
Competing Interest Statement

The authors have declared no competing interest.

Received May 20, 2015; accepted in revised form August 27, 2015.

\section{Phylogenetic Analysis of $16 \mathrm{~S}$ rRNA Gene Sequencing Data}

Resulting Reads of Insert consensus sequences were analyzed in the release 115 of the SILVA database (Quast et al. 2013) using v2.2.29 of BLAST (Altschul et al. 1990). An e-value of 0.001 was imposed as threshold and 100 sequences were retained ('-evalue 0.001 -max_target_ seqs $100^{\prime}$ ). Output from the BLAST alignment was analyzed with v5.0 of the MEGAN program (Huson et al. 2007) with a threshold of at least five supporting reads for a taxonomic level to be reported as present. Bacterial diversities at the species level have been estimated by calculating Shannon indices (Etter 1999).

\section{ADDITIONAL INFORMATION}

\section{Ethics Statement}

Informed consent has been obtained from the study participants. This study was approved by the Institutional Review Board of the University Hospital Zurich.

\section{Database Deposition and Access}

The sequencing data have been deposited in the NCBI SRA database (http://www.ncbi.nlm. nih.gov/sra) under BioProject ID PRJNA292639 and SRA ID SRP062303. Accession numbers of individual samples are SRX1143098 (D0), SRX1142599 (P1), SRX1143096 (P2), SRX1143095 (P3), SRX1143099 (D4), and SRX1143097 (P4).

\section{Acknowledgments}

The authors thank the donor and patient for their cooperation and their strong support of this study. We are also grateful to Prof. Peter H. Seeberger, Max Planck Institute of Colloids and Interfaces, Potsdam, Germany, for generous support of F.B. We thank colleagues from the Max Planck Institute for Molecular Genetics, Berlin, Germany for their support of this work and access to laboratory infrastructures and in particular to the bioinformatics facilities. The excellent technical assistance of Monique Herensperger is gratefully acknowledged.

\section{Author Contributions}

F.B., J.K., and M.S. performed the laboratory experiments and coordinated the work with G.Ru. who generated the data at the Functional Genomics Centre, Zurich, Switzerland. M.H. was involved in guidance of the patient and the donor, G.Ro. and L.B. are the medical doctors responsible for the clinical and ethical aspects involved. K.M. initiated and coordinated the project. F.B., J.K., and K.M. wrote the manuscript with assistance from G.Ru.

\section{Funding}

We thank colleagues of the Institute of Medical Microbiology, University of Zurich, Switzerland and of the Institute of Food, Nutrition and Health, ETH Zurich, Zurich, Switzerland for their generous financial support. K.M. supplied some private funds.

\section{REFERENCES}

Altschul SF, Gish W, Miller W, Myers EW, Lipman DJ. 1990. Basic local alignment search tool. J Mol Biol 215: 403-410.

Barr JJ, Auro R, Furlan M, Whiteson KL, Erb ML, Pogliano J, Stotland A, Wolkowicz R, Cutting AS, Doran KS, et al. 2013. Bacteriophage adhering to mucus provide a non-host-derived immunity. Proc Natl Acad Sci 110: 10771-10776.

Benson DA, Karsch-Mizrachi I, Lipman DJ, Ostell J, Sayers EW. 2009. GenBank. Nucleic Acids Res 37: 377-386. 
Born Y, Fieseler L, Klumpp J, Eugster MR, Zurfluh K, Duffy B, Loessner MJ. 2014. The tail-associated depolymerase of Erwinia amylovora phage L1 mediates host cell adsorption and enzymatic capsule removal, which can enhance infection by other phage. Environ Microbiol 16: 2168-2180.

Broecker F, Kube M, Klumpp J, Schuppler M, Biedermann L, Hecht J, Hombach M, Keller PM, Rogler G, Moelling K. 2013. Analysis of the intestinal microbiome of a recovered Clostridium difficile patient after fecal transplantation. Digestion 88: 243-251.

Cammarota G, laniro G, Gasbarrini A. 2014. Fecal microbiota transplantation for the treatment of Clostridium difficile infection: a systematic review. J Clin Gastroenterol 48: 693-702.

Carneiro M, Russ C, Ross M, Gabriel S, Nusbaum C, DePristo M. 2012. Pacific Biosciences sequencing technology for genotyping and variation discovery in human data. BMC Genomics 13: 375.

Claesson MJ, Cusack S, O'Sullivan O, Greene-Diniz R, de Weerd H, Flannery E, Marchesi JR, Falush D, Dinan T, Fitzgerald G, et al. 2011. Composition, variability, and temporal stability of the intestinal microbiota of the elderly. Proc Natl Acad Sci 108(Suppl 1): 4586-4591.

Eckburg PB, Bik EM, Bernstein CN, Purdom E, Dethlefsen L, Sargent M, Gill SR, Nelson KE, Relman DA. 2005. Diversity of the human intestinal microbial flora. Science 308: 1635-1638.

Etter W. 1999. Community analysis. In Numerical palaeobiology (ed. Harper DAT), pp. 285-360. John Wiley and Sons, Chichester, UK.

Fichot EB, Norman RS. 2013. Microbial phylogenetic profiling with the Pacific Biosciences sequencing platform. Microbiome 1: 10.

Gough E, Shaikh H, Manges AR. 2011. Systematic review of intestinal microbiota transplantation (fecal bacteriotherapy) for recurrent Clostridium difficile infection. Clin Infect Dis 53: 994-1002.

Huson DH, Auch AF, Qi J, Schuster SC. 2007. MEGAN analysis of metagenomic data. Genome Res 17: 377-386.

Johnson S. 2009. Recurrent Clostridium difficile infection: a review of risk factors, treatments, and outcomes. J Infect 58: 403-410.

Joossens M, Huys G, Cnockaert M, De Preter V, Verbeke K, Rutgeerts P, Vandamme P, Vermeire S. 2011. Dysbiosis of the faecal microbiota in patients with Crohn's disease and their unaffected relatives. Gut 60: 631-637.

Lepage P, Colombet J, Marteau P, Sime-Ngando T, Doré J, Leclerc M. 2008. Dysbiosis in inflammatory bowel disease: a role for bacteriophages? Gut 57: 424-425.

Lessa FC, Gould CV, McDonald LC. 2012. Current status of Clostridium difficile infection epidemiology. Clin Infect Dis 55(Suppl 2): S65-S70.

Li A, Chu Y, Wang X, Ren L, Yu J, Liu X, Yan J, Zhang L, Wu S, Li S. 2013. A pyrosequencing-based metagenomic study of methane-producing microbial community in solid-state biogas reactor. Biotechnol Biofuels 6: 3 .

Lo Vecchio A, Zacur GM. 2012. Clostridium difficile infection: an update on epidemiology, risk factors, and therapeutic options. Curr Opin Gastroenterol 28: 1-9.

Marshall CW, Ross DE, Fichot EB, Norman RS, May HD. 2012. Electrosynthesis of commodity chemicals by an autotrophic microbial community. Appl Environ Microbiol 78: 8412-8420.

McDonald LC, Killgore GE, Thompson A, Owens RC Jr, Kazakova SV, Sambol SP, Johnson S, Gerding DN. 2005. An epidemic, toxin gene-variant strain of Clostridium difficile. N Engl J Med 353: 2433-2441.

Minot S, Bryson A, Chehoud C, Wu GD, Lewis JD, Bushman FD. 2013. Rapid evolution of the human gut virome. Proc Natl Acad Sci 110: 12450-12455.

Miquel S, Martín R, Rossi O, Bermúdez-Humarán LG, Chatel JM, Sokol H, Thomas M, Wells JM, Langella P. 2013. Faecalibacterium prausnitzii and human intestinal health. Curr Opin Microbiol 16: 255-261.

Norman JM, Handley SA, Baldridge MT, Droit L, Liu CY, Keller BC, Kambal A, Monaco CL, Zhao G, Flehner P, et al. 2015. Disease-specific alterations in the enteric virome in inflammatory bowel disease. Cell 160: $447-460$.

Ogilvie LA, Caplin J, Dedi C, Diston D, Cheek E, Bowler L, Taylor H, Ebdon J, Jones BV. 2012. Comparative (meta)genomic analysis and ecological profiling of human gut-specific bacteriophage $\phi B 124-14$. PLoS One 7: e35053.

O'Horo JC, Jindai K, Kunzer B, Safdar N. 2014. Treatment of recurrent Clostridium difficile infection: a systematic review. Infection 42: 43-59.

Phan TG, Kapusinszky B, Wang C, Rose RK, Lipton HL, Delwart EL. 2011. The fecal viral flora of wild rodents. PLoS Pathog 7: e1002218.

Quail MA, Smith M, Coupland P, Otto TD, Harris SR, Connor TR, Bertoni A, Swerdlow HP, Gu Y. 2012. A tale of three next generation sequencing platforms: comparison of Ion Torrent, Pacific Biosciences and Illumina MiSeq sequencers. BMC Genomics 13: 341.

Quast C, Pruesse E, Yilmaz P, Gerken J, Schweer T, Yarza P, Peplies J, Glöckner FO. 2013. The SILVA ribosomal RNA gene database project: improved data processing and web-based tools. Nucleic Acids Res 41 (Database issue): D590-D596. 
COLD SPR ING HARB OR Molecular Case Studies
Long-term human microbiota after fecal transplant

Razavi B, Apisarnthanarak A, Mundy LM. 2007. Clostridium difficile: emergence of hypervirulence and fluoroquinolone resistance. Infection 35: 300-307.

Salzberg SL, Delcher AL, Kasif S, White O. 1998. Microbial gene identification using interpolated Markov models. Nucleic Acids Res 26: 544-548.

Sharpton TJ. 2014. An introduction to the analysis of shotgun metagenomic data. Front Plant Sci 5: 209.

Sokol H, Pigneur B, Watterlot L, Lakhdari O, Bermúdez-Humarán LG, Gratadoux JJ, Blugeon S, Bridonneau C, Furet JP, Corthier G, et al. 2008. Faecalibacterium prausnitzii is an anti-inflammatory commensal bacterium identified by gut microbiota analysis of Crohn's disease patients. Proc Natl Acad Sci 105: 16731-16736.

Thomas T, Gilbert J, Meyer F. 2012. Metagenomics-a guide from sampling to data analysis. Microb Inform Exp 2: 3.

Valverde JR, Mellado RP. 2013. Analysis of metagenomic data containing high biodiversity levels. PLoS One 8: e58118.

Van Etten JL. 2003. Unusual life style of giant chlorella viruses. Annu Rev Genet 37: 153-195.

van Nood E, Vrieze A, Nieuwdorp M, Fuentes S, Zoetendal EG, de Vos WM, Visser CE, Kuijper EJ, Bartelsman JF, Tijssen JG, et al. 2013. Duodenal infusion of donor feces for recurrent Clostridium difficile. N Engl J Med 368: 407-415.

Virgin HW. 2014. The virome in mammalian physiology and disease. Cell 157: 142-150.

Warny M, Pepin J, Fang A, Killgore G, Thompson A, Brazier J, Frost E, McDonald LC. 2005. Toxin production by an emerging strain of Clostridium difficile associated with outbreaks of severe disease in North America and Europe. Lancet 366: 1079-1084.

Weingarden A, González A, Vázquez-Baeza Y, Weiss S, Humphry G, Berg-Lyons D, Knights D, Unno T, Bobr A, Kang J, et al. 2015. Dynamic changes in short- and long-term bacterial composition following fecal microbiota transplantation for recurrent Clostridium difficile infection. Microbiome 3: 10.

Yolken RH, Jones-Brando L, Dunigan DD, Kannan G, Dickerson F, Severance E, Sabunciyan S, Talbot CC Jr, Prandovszky E, Gurnon JR, et al. 2014. Chlorovirus ATCV-1 is part of the human oropharyngeal virome and is associated with changes in cognitive functions in humans and mice. Proc Natl Acad Sci 111: $16106-16111$.

Youngster I, Russell GH, Pindar C, Ziv-Baran T, Sauk J, Hohmann EL. 2014. Oral, capsulized, frozen fecal microbiota transplantation for relapsing Clostridium difficile infection. JAMA 312: 1772-1778.

Zoetendal EG, Akkermans AD, de Vos WM. 1998. Temperature gradient gel electrophoresis analysis of 16S rRNA from human fecal samples reveals stable and host-specific communities of active bacteria. Appl Environ Microbiol 64: 3854-3859. 


\section{COLD SPRING HARBOR Molecular Case Studies}

\section{Long-term changes of bacterial and viral compositions in the intestine of a recovered Clostridium difficile patient after fecal microbiota transplantation}

Felix Broecker, Jochen Klumpp, Markus Schuppler, et al.

Cold Spring Harb Mol Case Stud 2016, 2: a000448 originally published online September 24, 2015 Access the most recent version at doi: $10.1101 / \mathrm{mcs}$.a000448

References This article cites 42 articles, 11 of which can be accessed free at: http://molecularcasestudies.cshlp.org/content/2/1/a000448.full.html\#ref-list-1

License This article is distributed under the terms of the Creative Commons Attribution License, which permits unrestricted reuse and redistribution provided that the original author and source are credited.

Email Alerting Receive free email alerts when new articles cite this article - sign up in the box at the Service top right corner of the article or click here. 